PROCEEDINGS OF THE

AMERICAN MATHEMATICAL SOCIETY

Volume 126, Number 10, October 1998, Pages 2841-2844

S 0002-9939(98)04392-5

\title{
ON THE CLASS EQUATION FOR HOPF ALGEBRAS
}

\author{
MARTIN LORENZ \\ (Communicated by Ken Goodearl)
}

\begin{abstract}
We give a simple proof of the Kac-Zhu class equation for semisimple Hopf algebras over an algebraically closed field of characteristic 0 .
\end{abstract}

\section{INTRODUCTION}

The class equation $([\mathrm{K}]$, Theorem $2,[\mathrm{Z}]$, Theorem 1 ) expresses a fundamental arithmetic property of semisimple Hopf algebras over an algebraically closed field of characteristic 0. It has featured prominently in many classification results for semisimple Hopf algebras of small or otherwise restricted dimension, notably in the work of Masuoka (cf. $[\mathrm{M}]$ and the references therein). All proofs heretofore ([K], $[\mathrm{S}]$ ) ultimately depend on the finiteness of the class number of algebraic number fields. The purpose of this short note is to give a simpler, more representation theoretic proof whose main ingredient is the known explicit form of the central primitive idempotents in split semisimple algebras.

In $\S 2$, we gather the requisite background material and give the statement of the class equation. The reader is referred to $[\mathrm{L}]$ or $[\mathrm{S}]$ for complete details. $\S 3$ then presents our proof of the class equation.

Throughout, $H$ denotes a finite dimensional Hopf algebra over a field $k$ of characteristic $p \geq 0$, with augmentation $\varepsilon$ and antipode $\mathcal{S}$. All $H$-modules are assumed to be finite dimensional left modules.

\section{THE ClASS EQUATION}

2.1. Character algebra and Grothendieck ring. The character algebra $R(H)$ of $H$ is the $k$-subalgebra of the dual Hopf algebra $H^{*}$ that is generated by the characters $\chi_{V}$ of $H$-modules $V$. A $\mathbb{Z}$-form of $R(H)$ is provided by the Grothendieck ring $G_{0}(H)$, the abelian group generated by the isomorphism classes $[V]$ of $H$ modules $V$ modulo the relations $[V]=[U]+[W]$ for each short exact sequence $0 \rightarrow U \rightarrow V \rightarrow W \rightarrow 0$. Multiplication in $G_{0}(H)$ is given by $[V] \cdot[W]=\left[V \otimes_{k} W\right]$. The character map $\chi: G_{0}(H) \rightarrow R(H),[V] \mapsto \chi_{V}$, induces an isomorphism of $k$-algebras

$$
R(H) \cong G_{0}(H) \otimes_{\mathbb{Z}} k
$$

Received by the editors December 16, 1996 and, in revised form, March 13, 1997.

1991 Mathematics Subject Classification. Primary 16W30; Secondary 16G10.

Key words and phrases. Hopf algebra, Grothendieck ring, character algebra, idempotent. Research supported in part by NSF Grant DMS-9400643.

(C)1998 American Mathematical Society 
Putting $[V]^{*}=\left[V^{*}\right]$, where $V^{*}=\operatorname{Hom}_{k}(V, k)$, one obtains an anti-automorphism * of the ring $G_{0}(H)$. Similarly, the antipode $\mathcal{S}^{*}$ of $H^{*}$ stabilizes $R(H)$ and thus yields an anti-automorphism of $R(H)$. The character map respects these antiautomorphisms:

$$
\chi_{V^{*}}=S^{*}\left(\chi_{V}\right) \text {. }
$$

We will fix a complete set of simple $H$-modules $V_{1}, \ldots, V_{t}$, with $V_{1}=k_{\varepsilon}$ the "trivial" $H$-module, and we put $\chi_{i}=\chi_{V_{i}}$, the irreducible characters of $H$. Then $\left\{\left[V_{1}\right], \ldots,\left[V_{t}\right]\right\}$ is a $\mathbb{Z}$-basis of $G_{0}(H)$ and $\left\{\chi_{1}, \ldots, \chi_{t}\right\}$ is a $k$-basis of $R(H)$, with $\left[V_{1}\right]=\left[k_{\varepsilon}\right]$ and $\chi_{1}=\varepsilon$ the respective identity elements of $G_{0}(H)$ and $R(H)$.

Examples. (1) $H=k G$, the group algebra of a finite group $G$ over a splitting field $k$. The dual Hopf algebra $H^{*}$ is the algebra of $k$-valued functions on $G$, $H^{*}=k^{G}$, with pointwise algebra operations, and $R(H)$ is the subalgebra of all $k$-valued functions $f$ on $G$ that are constant on the conjugacy classes of $G$ and satisfy $f(g)=f\left(g_{p^{\prime}}\right)$ for all $g \in G$. Here, $g_{p^{\prime}}$ denotes the $p$-regular part of $g$, that is, the component of $g$ whose order is not divisible by $p=$ char $k$. So

$$
R(H) \cong k^{T\left(G_{p^{\prime}}\right)}
$$

where $T\left(G_{p^{\prime}}\right)$ denotes the set of $G$-conjugacy classes of $p$-regular elements of $G$. Of course, if $p$ does not divide the order of $G$ then $T\left(G_{p^{\prime}}\right)$ is the set of all conjugacy classes of $G$. Furthermore, via Brauer characters, $G_{0}(H) \subseteq G_{0}(H) \otimes_{\mathbb{Z}} \mathbb{C} \cong \mathbb{C}^{T\left(G_{p^{\prime}}\right)}$.

(2) $H=(k G)^{*}$, the dual Hopf algebra of $k G$. Here, $R(H)=H^{*}=k G$ and $G_{0}(H)=\mathbb{Z} G$.

2.2. The semisimple case. Now assume $H$ split semisimple. Then $G_{0}(H)$ is a semiprime ring and $*$ is an involution (cf. [L], Proposition 3.2 and Remark 2.5(2)). The form $\langle.,\rangle:. G_{0}(H) \times G_{0}(H) \rightarrow \mathbb{Z}$ that is defined by

$$
\langle[V],[W]\rangle=\operatorname{dim}_{k} \operatorname{Hom}_{H}\left(V, W^{*}\right)
$$

is bilinear, associative, symmetric, and $*$-invariant $([\mathrm{L}], \S 3.1)$. A pair of dual $\mathbb{Z}$-bases of $G_{0}(H)$ with respect to $\langle.,$.$\rangle is given by \left\{\left[V_{1}\right], \ldots,\left[V_{t}\right]\right\}$ and $\left\{\left[V_{1}\right]^{*}, \ldots,\left[V_{t}\right]^{*}\right\}$ : $\left\langle\left[V_{i}\right],\left[V_{j}\right]^{*}\right\rangle=\delta_{i, j}$.

By $k$-linear extension to $R(H) \cong G_{0}(H) \otimes_{\mathbb{Z}} k$, one obtains a form $\beta: R(H) \times$ $R(H) \rightarrow k$ with analogous properties. In particular, $R(H)$ is a symmetric algebra, and $\left\{\chi_{1}, \ldots, \chi_{t}\right\},\left\{\mathcal{S}^{*}\left(\chi_{1}\right), \ldots, \mathcal{S}^{*}\left(\chi_{t}\right)\right\}$ is a pair of dual bases for $\beta$. If $p=$ char $k=$ 0 , then $R(H)$ is semisimple, as a consequence of semiprimeness of $G_{0}(H)$.

2.3. The Class Equation (Kac-Zhu). Assume $H$ semisimple and $k$ algebraically closed of characteristic $p=0$. Then, for every primitive idempotent $e=e^{2} \in R(H)$, $\operatorname{dim}_{k} e H^{*}$ divides $\operatorname{dim}_{k} H$.

Examples. For $H=k G$, the class equation reduces to the fact that the size of each conjugacy class of $G$ divides $|G|$. For $H=(k G)^{*}$, it says that the dimension of each simple $k G$-module divides $|G|$ (Frobenius' Theorem).

\section{The PROOF}

Letting $A$ denote the ring of algebraic integers in $k$, it suffices to show that

$$
d:=\frac{\operatorname{dim}_{k} H}{\operatorname{dim}_{k} e H^{*}} \in A
$$


To this end, we first express $d$ in terms of the central primitive idempotent $\widehat{e} \in$ $\mathcal{Z}(R(H))$, the center of $R(H)$, with $e=e \widehat{e}$. In other words, $\widehat{e} R(H)$ is the simple component of the split semisimple $k$-algebra $R(H)$ containing $e$. Thus, if $m$ denotes the dimension of the irreducible $R(H)$-module $e R(H)$, then $\widehat{e} R(H) \cong(e R(H))^{m}$. This implies $\widehat{e} H^{*} \cong\left(e H^{*}\right)^{m}$, and hence

$$
d=\frac{m \operatorname{dim}_{k} H}{\operatorname{dim}_{k} \widehat{e} H^{*}} .
$$

Central primitive idempotents can be expressed in terms of dual bases (cf. [CR], Proposition (9.17)). In our situation, in view of $\S 2.2$, this expression takes the following form:

$$
\widehat{e}=\omega(\widetilde{e})^{-1} \widetilde{e} \quad \text { with } \quad \widetilde{e}=\sum_{i} \mu\left(\mathcal{S}^{*}\left(\chi_{i}\right)\right) \chi_{i},
$$

where $\omega: \mathcal{Z}(R(H)) \rightarrow k$ denotes the central character of the irreducible $R(H)$ module $e R(H)$ and $\mu$ its (ordinary) character; so $m=\mu(\varepsilon)$ and $\omega(z)=m^{-1} \mu(z)$. We claim that

$$
d=\omega(\widetilde{e}) .
$$

Indeed, defining $L_{f} \in \operatorname{End}_{k}\left(H^{*}\right)$ by $L_{f}(g)=f g$ for $f, g \in H^{*}$, the element $x=$ $\left(f \mapsto \operatorname{trace}\left(L_{f}\right)\right) \in H^{* *}=H$ is an integral of $H$ ([LS], Proposition 9; recall that $H$ is involutory, by $[\mathrm{LR}])$. Since $\widehat{e}$ is idempotent, we have $\operatorname{dim}_{k} \widehat{e} H^{*}=\operatorname{trace}\left(L_{\widehat{e}}\right)$, and so

$$
\operatorname{dim}_{k} \widehat{e} H^{*}=\widehat{e}(x)=\omega(\widetilde{e})^{-1} \widetilde{e}(x) .
$$

Finally, $\chi_{1}(x)=x(\varepsilon)=\operatorname{dim}_{k} H^{*}=\operatorname{dim}_{k} H$ and $\chi_{i}(x)=0$ for $i>1$, since $x V_{i}$ consists of $H$-invariants in $V_{i}$ and hence must vanish. Thus,

$$
\widetilde{e}(x)=\mu(\varepsilon) \operatorname{dim}_{k} H=m \operatorname{dim}_{k} H
$$

and our claim follows.

To complete the proof, we remark that if $\phi$ is any character of $R(H)$, then, identifying $G_{0}(H)$ with its image in $R(H)$ under the character map, we have $\phi\left(G_{0}(H)\right) \subseteq A$. For, any $\alpha \in G_{0}(H)$ satisfies a monic polynomial over $\mathbb{Z}$, and hence so does the image $\Phi(\alpha)$ under the representation $\Phi$ belonging to $\phi$, and all eigenvalues of $\Phi(\alpha)$ as well. Thus, $\phi(\alpha)=\operatorname{trace}(\Phi(\alpha)) \in A$. In particular, $\widetilde{e}=\sum_{i} \mu\left(\mathcal{S}^{*}\left(\chi_{i}\right)\right) \chi_{i}$ belongs to $G_{0}(H) \otimes_{\mathbb{Z}} A$. Hence $\widetilde{e}$ is integral over $\mathbb{Z}$ and, therefore, so is $d=\omega(\widetilde{e})$.

\section{REFERENCES}

[CR] C. W. Curtis and I. Reiner, Methods of Representation Theory, Vol. 1, Wiley-Interscience, New York, 1981. MR 90k:20001

[K] G. I. Kac, Certain arithmetic properties of ring groups, Functional Anal. Appl. 6 (1972), 158-160. MR 46:3687

[LR] R. G. Larson and D. E. Radford, Semisimple cosemisimple Hopf algebras, Amer. J. Math. 109 (1987), 187-195. MR 89a:16011

[LS] R. G. Larson and M. E. Sweedler, An associative orthogonal bilinear form for Hopf algebras, Amer. J. Math. 91 (1969), 75-94. MR 39:1523

[L] M. Lorenz, Representations of finite dimensional Hopf algebras, J. Algebra 188 (1997), 476-505. CMP 97:08

[M] A. Masuoka, Some further classification results of semisimple Hopf algebras, Commun. Algebra 24 (1996), 307-329. MR 96k:16070 
[S] H.-J. Schneider, Lectures on Hopf Algebras, Lecture Notes, Universidad Nacional de Cordoba, 1995.

[Z] Y. Zhu, Hopf algebras of prime dimension, Internat. Math. Res. Notices 1 (1994), 53-59. MR 94j:16072

Department of Mathematics, Temple University, Philadelphia, Pennsylvania 191226094

E-mail address: lorenz@math.temple.edu 